HU-EP-07/09

DESY-07-043

MIT-CTP 3827

\title{
Area-preserving diffeomorphisms in gauge theory on a non-commutative plane: a lattice study
}

\author{
Wolfgang Bietenholz ${ }^{\mathrm{a}}$, Antonio Bigarini ${ }^{\mathrm{b}, \mathrm{c}}$ and Alessandro Torrielli ${ }^{\mathrm{d}}$ \\ a John von Neumann Institut für Computing (NIC) \\ Deutsches Elektron Sychrotron (DESY) \\ Platanenallee 6, D-15738 Zeuthen, Germany \\ b Dipartimento di Fisica, Università degli Studi di Perugia \\ and INFN, Sezione di Perugia, \\ Via Pascoli 1, I-06100 Perugia, Italy \\ ${ }^{c}$ Institut für Physik \\ Humboldt-Universität zu Berlin \\ Newtonstr. 15, D-12489 Berlin, Germany \\ ${ }^{\mathrm{d}}$ Center for Theoretical Physics, Laboratory for Nuclear Sciences \\ and Department of Physics \\ Massachusetts Institute of Technology \\ 77 Massachusetts Avenue, Cambridge, MA 02139-4307, USA
}

\begin{abstract}
We consider Yang-Mills theory with the $U(1)$ gauge group on a non-commutative plane. Perturbatively it was observed that the invariance of this theory under area-preserving diffeomorphisms (APDs) breaks down to a rigid subgroup $S L(2, R)$. Here we present explicit results for the APD symmetry breaking at finite gauge coupling and finite non-commutativity. They are based on lattice simulations and measurements of Wilson loops with the same area but with a variety of different shapes. Our results are consistent with the expected loss of invariance under APDs. Moreover, they strongly suggest that non-perturbatively the $S L(2, R)$ symmetry does not persist either.
\end{abstract}




\section{Introduction}

Invariance under area-preserving diffeomorphisms (APDs) [1] is a basic symmetry of ordinary Yang-Mills theories in two dimensions. In particular it means that Wilson loop expectation values only depend on the oriented areas singled out on the manifold. Thanks to this property the theory acquires an almost topological flavour [2] and, as a consequence, it can be solved analytically. Elegant group theoretic methods [3] lead to closed expressions for the partition function and a set of observables [4].

The invariance under APDs was initially believed to persist also in $U(n)$ gauge theories defined on a non-commutative (NC) two-dimensional manifold. It was assumed to play a central role in the large gauge group characteristic of gauge theories on NC spaces - which merges internal and space-time transformations. A detailed study of the non-commutative gaugetransformation algebra was performed in Ref. [5]. If APD symmetry holds, one might hope to be able to solve gauge theories also on a $\mathrm{NC}$ plane by generalising the powerful geometric procedures developed in the commutative space.

This scenario was suggested by an intriguing observation for $U(n)$ gauge theory on a $\mathrm{NC}$ torus. It can be related by Morita equivalence to its dual on a commutative torus [6], where the APD invariance is granted. The theory on the NC plane would then be reached by a suitable limit, and one could hope for the invariance to be preserved [7].

Wilson loop perturbative expansions in the coupling constant $g$ and in $1 / \theta$ - $\theta$ being the non-commutativity parameter - were performed on the $\mathrm{NC}$ plane. To the order first considered in Refs. $[8,9]$, the results were consistent with APD invariance.

Later on Ref. [10] extended those results to the next order, namely $\mathcal{O}\left(\theta^{-2}\right)$ at $\mathcal{O}\left(g^{4}\right)$. The outcome revealed different expectation values for a Wilson loop with the shape of a circle and a rectangle of the same area. This observation motivated the systematic investigation in Ref. [11], where Wilson loops in a wide class of contours were considered in the axial gauge. These results suggest that the APD symmetry breaks down to a residual subgroup of linear unimodular transformations, $S L(2, R)$.

Subsequently non-perturbative arguments for this APD symmetry breaking were given based on the Morita duality on tori [12]. Recently, Ref. [13] reconsidered this issue by applying twist deformation techniques, which also confirm that the APD symmetry may break at the quantum level. A new study of the large $\theta$ expansion [14] reports the break-down of the area law at $\mathcal{O}\left(1 / \theta^{2}\right)$, in agreement with simulations results at finite $\theta$ [15]. Ref. [14] does not report the observation of any symmetry. However, the question about 
the ultimate status of (partial) APD symmetry is still open.

The present work presents explicit results for Wilson loops with polygonal contours, at finite $\theta$ and $g$, under APDs. Our non-perturbative results are obtained on the lattice and extrapolated to the continuum. They agree with the breaking of this symmetry, both on the lattice and in the continuum limit. Moreover we provide evidence against the survival of a residual symmetry subgroup $S L(2, R)$.

In Section 2 we briefly review the $U(1)$ gauge theory on a $\mathrm{NC}$ plane, its lattice discretisation and the mapping onto a twisted Eguchi-Kawai model, which can be simulated. Section 3 presents our simulation results for the planar limit, which is necessary to identify a physical scale. Then we address in Section 4 the Double Scaling Limit to a continuous NC plane of infinite extent, which allows us to study explicitly the effect of APD transformations on Wilson loops. In Section 5 we confirm the APD symmetry breaking by considering observables, which differ from those in Section 4. Their perturbative treatment is commented on in an Appendix. Section 6 focuses specifically on the $S L(2, R)$ symmetry, and Section 7 is dedicated to our conclusions.

\section{$2 \quad U(1)$ gauge theory on a non-commutative plane}

In this work, we consider the simplest version of a Euclidean NC plane by assuming a constant non-commutativity parameter $\theta$, so that the coordinate operators fulfil

$$
\left[\hat{x}_{\mu}, \hat{x}_{\nu}\right]=\mathrm{i} \theta \epsilon_{\mu \nu} \quad(\mu, \nu=1,2) .
$$

Such coordinates describe a charged particle moving in a (commutative) plane, which is crossed by a strong, orthogonal magnetic field. The latter can be formally interpreted as $B \propto 1 / \theta$, see e.g. Ref. [16]. A similar concept is also used to map open strings in a magnetic background onto $\mathrm{NC}$ field theory [17].

We can return to the use of ordinary (commutative) coordinates if all the fields are multiplied by star products (or Moyal products),

$$
\phi(x) \star \psi(x):=\phi(x) \exp \left(\frac{\mathrm{i}}{2} \overleftarrow{\partial}_{\mu} \theta \epsilon_{\mu \nu} \vec{\partial}_{\nu}\right) \psi(x)
$$

Here we focus on pure $U(1)$ gauge theory with the Euclidean action

$$
\begin{aligned}
S[A] & =\frac{1}{4} \int d^{2} x F_{\mu \nu} \star F_{\mu \nu}, \\
F_{\mu \nu} & =\partial_{\mu} A_{\nu}-\partial_{\nu} A_{\mu}+i g\left[A_{\mu}, A_{\nu}\right]_{\star} .
\end{aligned}
$$


The last term is a star-commutator, which shows that even the $U(1)$ gauge field is self-interacting on NC spaces. This action is star-gauge invariant, i.e. invariant under transformations

$$
A_{\mu}(x) \rightarrow U(x) \star A_{\mu}(x) \star U(x)^{\dagger}-\frac{\mathrm{i}}{g} U(x) \star \partial_{\mu} U(x)^{\dagger},
$$

if $U(x)$ is star-unitary, $U(x)^{\dagger} \star U(x)=\mathbb{1}$.

Other $U(n)$ gauge theories may be studied along the same lines, but the formulation of $S U(n)$ gauge theories runs into trouble on NC spaces. Therefore it is motivated to concentrate on $U(1)$ as a physical gauge group, which can be accommodated on NC manifolds.

Although the points in such spaces are somewhat fuzzy, it is possible to introduce a lattice structure 1 This is a first step towards a formulation to be used in Monte Carlo simulations. In the operator formalism this step imposes the constraint

$$
\exp \left(\mathrm{i} \frac{2 \pi}{a} \hat{x}_{\mu}\right)=\hat{\mathbb{1}}
$$

where $a$ is the lattice spacing. If we require the momentum components to be commutative and periodic over the Brillouin zone, the above condition implies that only discrete momenta occur, which is characteristic for a finite volume. On a $N \times N$ lattice with periodic boundary conditions, the allowed momenta are spaced by $2 \pi /(a N)$. As a consequence, the non-commutativity parameter can be identified as

$$
\theta=\frac{1}{\pi} N a^{2}
$$

We are most interested in a Double Scaling Limit (DSL)

$$
a \rightarrow 0 \text { and } N \rightarrow \infty \quad \text { at } \quad N a^{2}=\text { const. }
$$

which leads to a continuous NC plane of infinite extent. The requirement to take the UV and IR limits simultaneously in a balanced way is related to the generic UV/IR mixing of the divergences in NC field theory [20].

This is clearly distinct from the planar limit, $N \rightarrow \infty$ at fixed gauge coupling, which means here a fixed lattice spacing. The non-commutativity parameter diverges in this limit. In higher dimensions, this implies that nonplanar contributions are suppressed, and the planar limit restores commutativity in perturbation theory 2 In two dimensions the situation is different,

\footnotetext{
${ }^{1}$ Here we only sketch this regularisation very briefly, for details we refer for instance to the review [18], or the theses quoted in Refs. [15] and [19].

${ }^{2}$ We remark, however, that this restoration does not need to hold generally: it can fail non-perturbatively for instance in the case of spontaneous symmetry breaking [21].
} 
and non-planar diagrams provide "anomalous" perturbative contributions in the limit of infinite non-commutativity, which are of the same order of magnitude as the planar diagrams [8-10]. However, such terms are shown to disappear when applying the procedure recently introduced in Ref. [14].

Even on the lattice it is far from obvious how to simulate NC gauge theory; note that the compact formulation seems to require star-unitary link variables. In this respect, it is highly profitable to map the system onto a twisted Eguchi-Kawai model (TEK model). This model is defined on a single space-point and its action takes the form [22]

$$
S_{\mathrm{TEK}}[U]=-N \beta \sum_{\mu \neq \nu} Z_{\mu \nu} \operatorname{Tr}\left(U_{\mu} U_{\nu} U_{\mu}^{\dagger} U_{\nu}^{\dagger}\right)
$$

$U_{1}$ and $U_{2}$ are unitary $N \times N$ matrices which encode the degrees of freedom of the $U(1)$ lattice gauge theory. For the twist factor we adopt the choice of Ref. [15], $Z_{21}=Z_{12}^{*}=\exp (\mathrm{i} \pi(N+1) / N)$, where $N$ has to be odd. There is an exact equivalence to the lattice $\mathrm{NC} U(1)$ gauge theory, i.e. the algebras are identical, as Ref. [23] showed in the large $N$ limit. A refined consideration found such a mapping even at finite $N$ [24]. Hence the TEK model can be used for numerical simulations of NC gauge theories, and it is most suitable for this purpose.

It is straightforward to formulate Wilson loops in this matrix model. For instance, a rectangular loop with side lengths $a I$ and $a J$ (and clockwise orientation) corresponds to the term

$$
W(I \times J)=\frac{1}{N} Z_{12}^{I J} \operatorname{Tr}\left(U_{1}^{I} U_{2}^{J} U_{1}^{\dagger I} U_{2}^{\dagger J}\right) .
$$

Mapping this quantity back to the lattice leads in fact to a sensible definition of a Wilson loop in the NC gauge theory [25,26]. Such Wilson loops are complex in general $[9,15]$. The action (8) is real, however, since both orientations of the plaquettes are summed over, which is essential for the feasibility of numerical simulations.

Simulations of gauge theories with the standard Metropolis algorithm are notoriously inefficient. This also holds for the TEK model. Moreover the usual remedy - the application of the heat-bath algorithm - cannot be applied straightforwardly, because the dimensionally reduced action (8) is non-linear in the link variables. However, by introducing an auxiliary matrix field the action can be linearised [27], so that the heat-bath algorithm works and the model can indeed be simulated efficiently 3 This method allowed us

\footnotetext{
${ }^{3}$ This method has recently been extended to the $4 \mathrm{~d}$ model where two dimensions are
} 
to explore rather large systems of $N>100$. For the parameter sets $(N, \beta)$ that we investigated, we collected statistics of 1000 well thermalised and decorrelated configurations.

\section{The planar limit}

In the planar limit we obtain the $U(N \rightarrow \infty)$ lattice gauge theory on a commutative plane, which was solved by Gross and Witten [28]. In this limit they found an exact area law for the Wilson loops. In (dimensionless) lattice units it takes the form

$$
\langle W(I \times J)\rangle=\exp (-\sigma(\beta) I J), \quad \sigma(\beta)=\left\{\begin{array}{cc}
-\ln \beta & \beta \leq 1 / 2 \\
-\ln \left(1-\frac{1}{4 \beta}\right) & \beta \geq 1 / 2
\end{array} .\right.
$$

In terms of dimensional units the string tension $\sigma$ turns into an area, and it allows us therefore to identify a dimensional lattice spacing as

$$
a=\sqrt{\sigma(\beta)} .
$$

In these units the string tension is set to 1 in the planar limit.

Of course, we are ultimately interested in the DSL according to eq. (7). But to give it an explicit meaning we first have to identify a dimensional lattice spacing, i.e. we have to introduce a scale to interpret the lattice units. Relation (11) can be used for this purpose $[15,30]$, provided that the values of $N$, which are accessible to our simulations, do approximate the planar limit well (for the quantities of interest). Otherwise one would have to worry about finite $N$ artifacts distorting the physical interpretation of our results.

We verified this property first by checking the validity of the large $N$ Schwinger-Dyson equations. On the lattice, they relate Wilson loops of different shapes in the planar limit 4 An example for the corresponding contours is illustrated in Figure 1 (cf. first work in Ref. [22]). Indeed, we observe that our measurements for the two sides of this equation converge well as we increase $N$ to a magnitude of $\mathcal{O}(100)$ at fixed $\beta$, see Figure 2 5

The Eguchi-Kawai equivalence to the model solved by Gross and Witten also implies the validity of the APD symmetry in the planar limit. Again we testify if this symmetry can be observed to set in (approximately) for the

reduced; this is adequate for QED in a four dimensional space, composed of a commutative and an NC plane [19].

${ }^{4}$ This was the property that motivated the original construction of the dimensionally reduced matrix model (without twist factor) by Eguchi and Kawai [29].

${ }^{5} \mathrm{~A}$ variant of this result has been reported before in a proceeding contribution [31] and in a Ph.D. thesis [32]. Earlier observations in this context were given in Ref. [30]. 


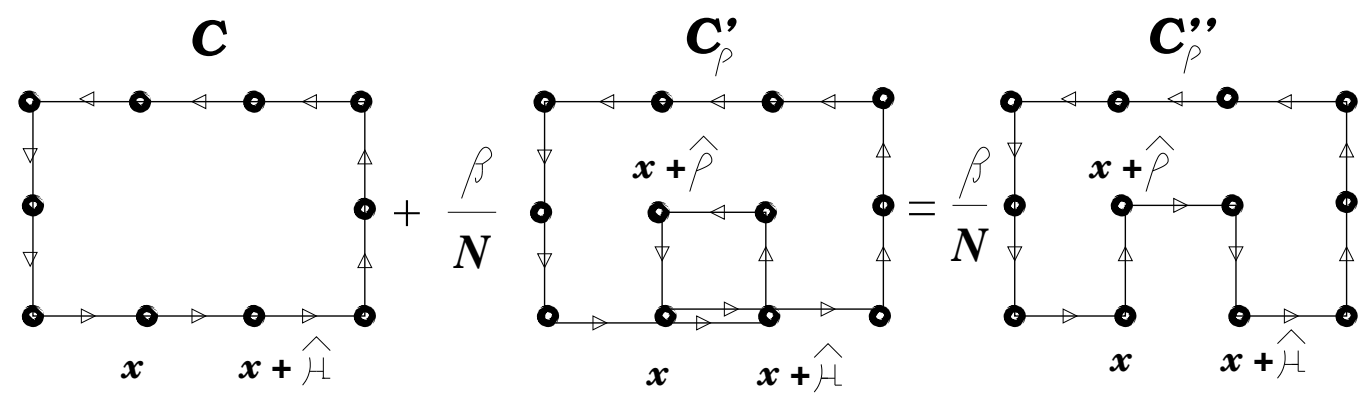

Figure 1: An example of a set of contours, which are involved in a SchwingerDyson equation. These equations relate the vacuum expectation values of the corresponding Wilson loops in the planar limit. They can be derived from the invariance under an infinitesimal substitution of the compact link variables on the lattice [22].
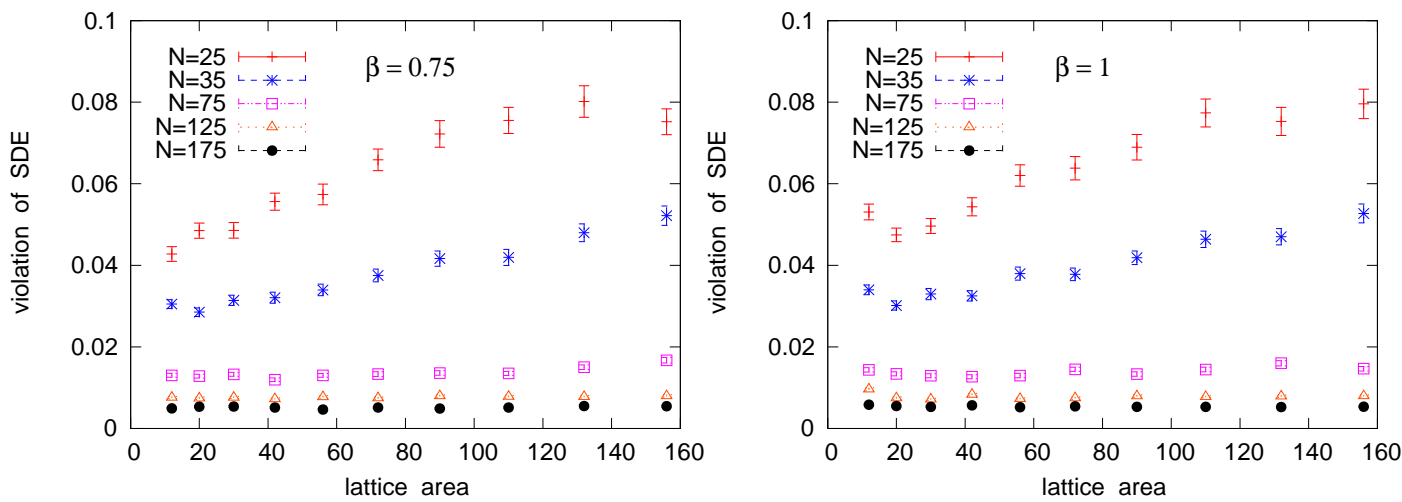

Figure 2: The convergence towards the validity of the Schwinger-Dyson equations as $N$ increases at fixed $\beta=0.75$ (on the left) and $\beta=1$ (on the right). The $y$-axis is the absolute value of the deviation between the two sides of the specific Schwinger-Dyson equation illustrated in Figure 1. The x-axis corresponds to the lattice area of the contour $C$, which has a rectangular shape of the form $I \times(I+1)$. These equations are well approximated as $N$ reaches $\mathcal{O}(100)$.

system sizes that we simulated. We consider four types of Wilson loops: we denote them as square loops, rectangular loops, stair loops and L-loops. In particular we considered these loops at the areas $A=4,9,16,25 \ldots$ in lattice units. The rectangular loops are maximally anisotropic, i.e. their shapes are rectangles of side lengths 1 and $A 6$ For the shapes of stair loops and L-loops we refer to Figure 3, Note that these two loop types involve two

\footnotetext{
${ }^{6}$ In Section 4 we will also consider rectangles with a fixed ratio between the two side lengths.
} 
slightly different cases, depending on whether the area $A$ is even or odd.
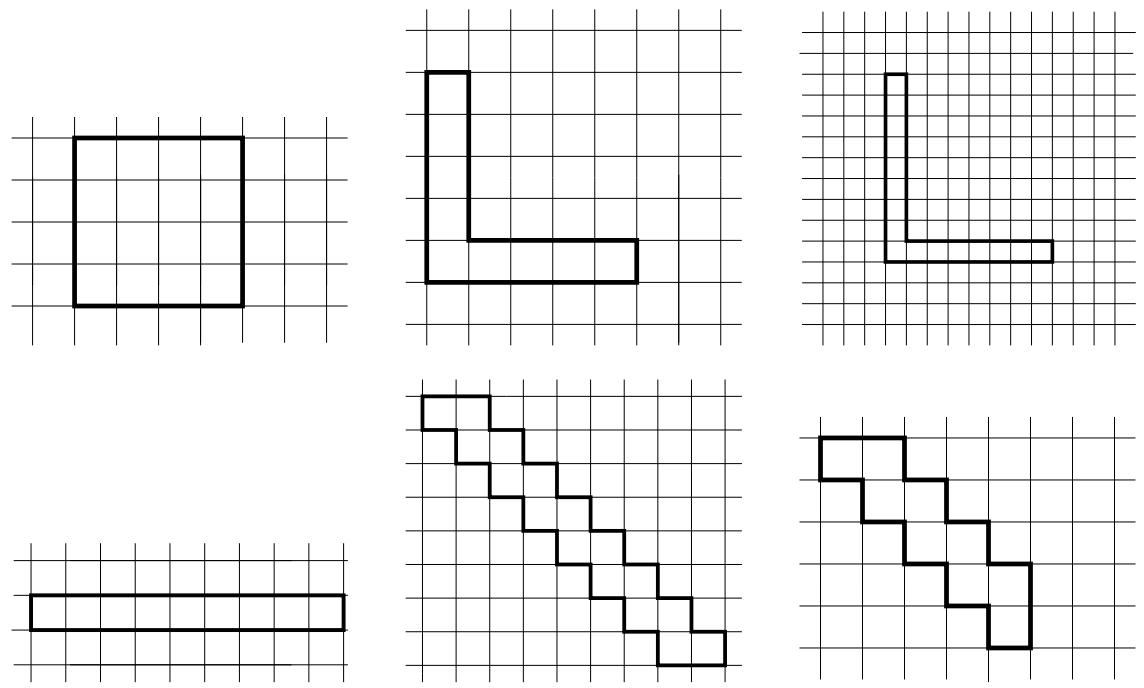

Figure 3: An illustration of the different Wilson loops that we considered. Their contours are all polygonal, without multiple-intersections. They are squares, L-shapes (with legs of width 1 lattice spacing and equal length, or lengths deviating by 1 if the area $A$ is even), maximally anisotropic rectangles and stairs (again in two variants, depending whether $A$ is even or odd).

All the four types of loops (with $A$ fixed) are related by APDs. In particular the square and rectangles transform into each other under $S L(2, R)$ on the plane.

We now present numerical results for these Wilson loops $W$ as we approach the planar limit. As an example, we first consider the absolute value $|W|$ as a function of the dimensional area at a fixed lattice area of $A=36$. Figure 4 shows results for $N=75$ and 155 . We see that the absolute values for the different shapes coincide for small and for large areas, but they split apart at an intermediate physical area of $A a^{2}=\mathcal{O}(10)$.

However, even in this intermediate regime the differences between the Wilson loops at a fixed area converge to zero in the planar limit; examples for this behaviour are shown in Figure 5. Since $\beta$ was kept fixed in these plots, both the area in lattice units and in dimensional units is constant.

For the interpretation of our simulation results, we can therefore rely on the scale (11) extracted from the planar limit. This allows us to proceed now to the investigation of the DSL, which describes the theory on a $\mathrm{NC}$ plane in the simultaneous UV and IR limit. 

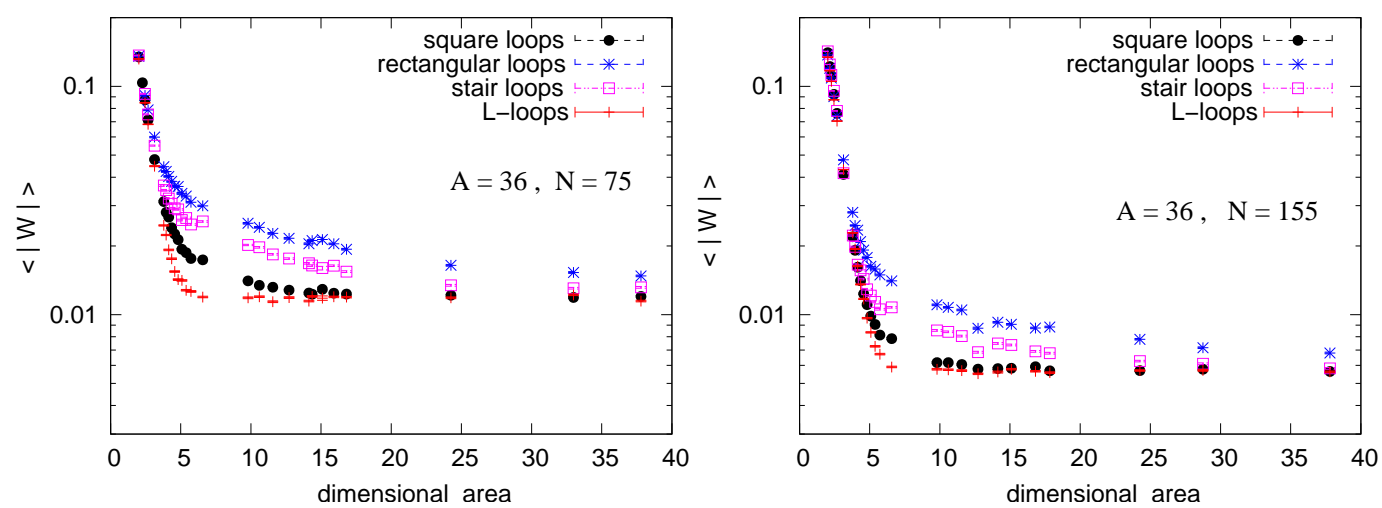

Figure 4: The absolute values of Wilson loops with different shapes and lattice area $A=36$. They are plotted against the physical area $36 a^{2}$, which is varied by using different values of $\beta$. We show results for $N=75$ on the left, and for $N=155$ on the right. The results for different shapes coincide best at small and at larger areas, but they differ most around $36 a^{2} \approx 10$.
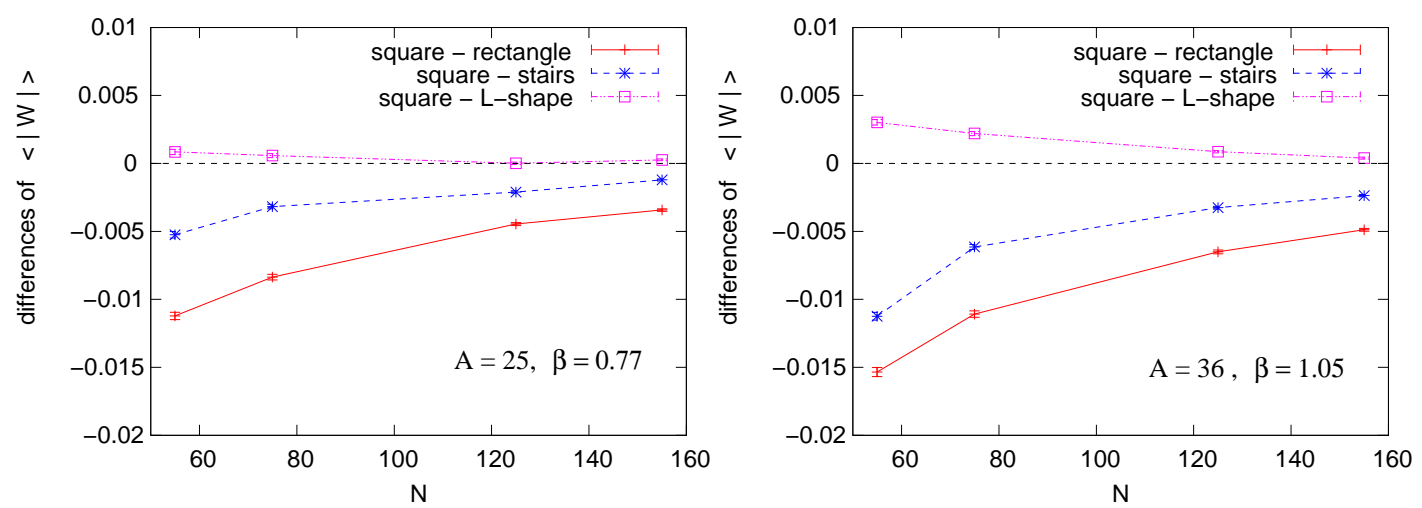

Figure 5: An illustration of the convergence towards the APD symmetry in the planar limit. On the left we show the differences of $\langle|W|\rangle$ for Wilson loops with lattice area $A=25$ at $\beta=0.77$, so the dimensional area amounts to $A a^{2} \simeq 9.81$. In the plot on the right-hand-side the parameters are $A=36$ and $\beta=1.05$, which implies almost the same area, $A a^{2} \simeq 9.79$. For increasing $N$ the differences between the Wilson loops with different shapes decrease rapidly, so we are approximating well the behaviour in the planar limit.

\section{The Double Scaling Limit}

We now employ the scale (11) provided by the Gross-Witten area law (10); its use for the range of parameters under consideration has been justified in Section 3. This enables us to study the DSL (17) to a continuous plane of infinite extent and finite non-commutativity. 
From Refs. [15] we know the following properties about the square loops:

- The observable $\langle W(I \times I)\rangle$ does indeed stabilise in the DSL. The existence of this universality class shows in particular that the model is non-perturbatively renormalisable.

- At small area, the absolute value $|\langle W(I \times I)\rangle|$ follows an area law. In that regime, which extends up to $(a I)^{2} \lesssim 4$, the phase is practically zero.

- For larger areas, $|\langle W(I \times I)\rangle|$ does not decay any further, but the phase starts to increase linearly in the area. It obeys the simple relation

$$
\text { phase }=\frac{(a I)^{2}}{\theta}=(a I)^{2} \cdot B,
$$

where we symbolically introduced a magnetic field $B=1 / \theta$ across the plane. As we mentioned in Section 2, this identification of the magnetic field has been implemented in string theory and in solid state physics. The behaviour (12) just corresponds to the Aharonov-Bohm effect, which fits perfectly into the magnetic interpretation of noncommutativity. Ref. [15] discovered this behaviour (unexpectedly) as a dynamical effect at low energy.

To revisit the second point - the area law for small Wilson loops, where the phase is practically zero - we measured the Creutz ratio

$$
\chi(I, J)=-\ln \left[\frac{\langle W(I \times J)\rangle\langle W((I-1) \times(J-1))\rangle}{\langle W((I-1) \times J)\rangle\langle W(I \times(J-1))\rangle}\right] .
$$

This ratio singles out the string tension $\sigma$ for decays $\propto \exp (-\sigma A)$, provided that it is equivalent for the various rectangular Wilson loops involved. Typical results for (nearly) square shaped Wilson loops, $\chi(I, I)$, as well as extremely anisotropic (rectangular) Wilson loops, $\chi(2, J)$, are shown in Figure 6 6 For both shapes we find a stable behaviour as we increase $N$ at fixed $\theta$, which suggests that our results can safely be extrapolated to the DSL. Deeply inside the area law regime we obtain $\sigma \simeq 1$. Hence in this range the behaviour in the DSL coincides with the planar limit. We observe, however, a marked deviation from it as the area approaches the transition to the regime of the Aharonov-Bohm type behaviour.

At moderate area the Creutz ratios for the squares and the rectangles differ a little. This is a first observation hinting at shape independence for rectangles deeply inside the area law regime, but not beyond.

\footnotetext{
${ }^{7}$ We actually averaged over $\chi(2, J)$ and $\chi(J, 2)$ in order to increase the statistics.
} 


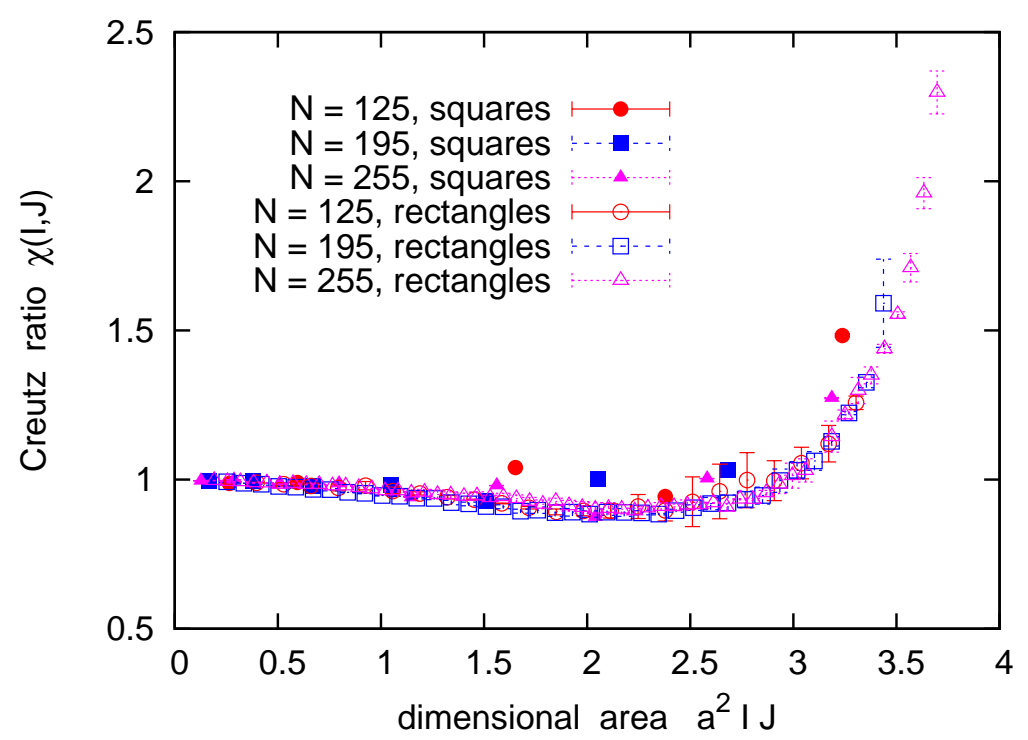

Figure 6: The Creutz ratio $\chi(I, J)$ for Wilson loops with small areas, up to the transition at the end of the area law regime. The parameter sets are $(N, \beta)=(125,3.91),(195,6.09)$ and $(255,7.97)$, which all corresponds to $\theta \approx 2.6$, so we are approaching a DSL. For both shapes, i.e. for $\chi(I, I)$ (squares) and for $\chi(2, J)$ (rectangles), we observe stable Creutz ratios in the DSL. At small area the string tension takes the same value as in the planar limit. At moderate area the results from square shaped and from extremely anisotropic rectangles begin to differ a little.

Regarding the behaviour at large areas, which is very specific to the NC plane, one may wonder why the short-ranged non-commutativity has striking effects on the large rather than the small Wilson loops. UV/IR mixing [20] is apparently at work, even though the perturbative expansion of this model can be formulated without divergences. This suggests that UV/IR mixing occurs non-perturbatively, and it belongs therefore to the fundamental nature of NC field theory. This is in agreement with analytic [33] and numerical $[32,34]$ results for the NC $\lambda \phi^{4}$ model, and for $4 \mathrm{~d}$ NC QED [19].

At last we proceed to a systematic study of the fate of the APD symmetry in the DSL. Figures 7 and 8 show results for the absolute values of Wilson loop expectation values, $|\langle W\rangle|$, with respect to the shapes illustrated in Figure 3. Figure 7 refers to a fixed non-commutativity parameter $\theta=1.63$, and it shows results for two values of $N$. For increasing $N$ the dimensional volume is enlarged and the lattice becomes finer (because $N a^{2}$ is kept constant), so 
we approach the DSL. The corresponding results at $\theta=2.63$ are presented in Figure 8. For the latter $\theta$ value we show in addition the Wilson loop phases $\arg (\langle W\rangle)$ in Figure 9. These figures demonstrate that the Wilson loops for $N=125$ and $N=155$ — plotted against the dimensional area - are almost identical. This shows that we are indeed in the asymptotic regime of the DSL. We can therefore be confident that our results reveal the behaviour in the continuous NC plane. This confidence will be further substantiated by results for variants of these observables to be presented in the Section 5 .

As soon as the area exceeds the area law regime, we observe a clear distinction between the absolute values $|\langle W\rangle|$ for different shapes, see Figures 7 and 8$]^{8}$ This distinction occurs for any pair of the contour types considered. We show again the rectangles of the form $1 \times A$ (in lattice units), which become infinitesimally narrow in the DSL (in dimensional units). In addition we also include rectangles with a fixed ratio of 4 between the side lengths, which keep an invariant shape as we approach the DSL. The different expectation values for the narrow rectangles with the same area show clearly that the APD symmetry breaks.

The distinction between squares and rectangles of fixed side ratio in these plots is less striking than the other cases. However, exactly these rectangles are directly relevant to explore the fate of the symmetry subgroup $S L(2, R)$, hence we will focus on them specifically in Section 6 .

Next we discuss the phases $\arg (\langle W\rangle)$, which we show for $\theta=2.63$ in Figure 9. As the area increases beyond the area law regime, the square shapes, rectangles with fixed side ratio and the stairs follow very well the Aharonov-Bohm type behaviour corresponding to eq. (12), which had been observed earlier for squares and certain rectangles [15]. The L-shape does not agree optimally, but its behaviour is reasonably close, in particular on the finer lattice which corresponds to $N=155$. We remark that the extremely anisotropic rectangles considered earlier lead to strong deviations from eq. (12), as we are going to show in the Section 5 (Figure 13). Apparently shapes which become extremely thin (in physical units) as we approach the DSL can lead to such features (although this is not the case for the stair loops).

In any case, our results for the phases are very similar for the different $N$ values, so they confirm that we are in an asymptotic window of the DSL. The phase of the Wilson loop has a much stronger trend towards (at least partial) APD symmetry than the absolute value. As far as we could check, the phase (alone) is well compatible with this symmetry for fixed shapes in dimensional units, with a finite extent in each direction. But of course the differences in

\footnotetext{
${ }^{8}$ We note that the total area of the system amounts to $V=\pi N \theta$, hence for our parameters $V \gg \theta$ is granted.
} 

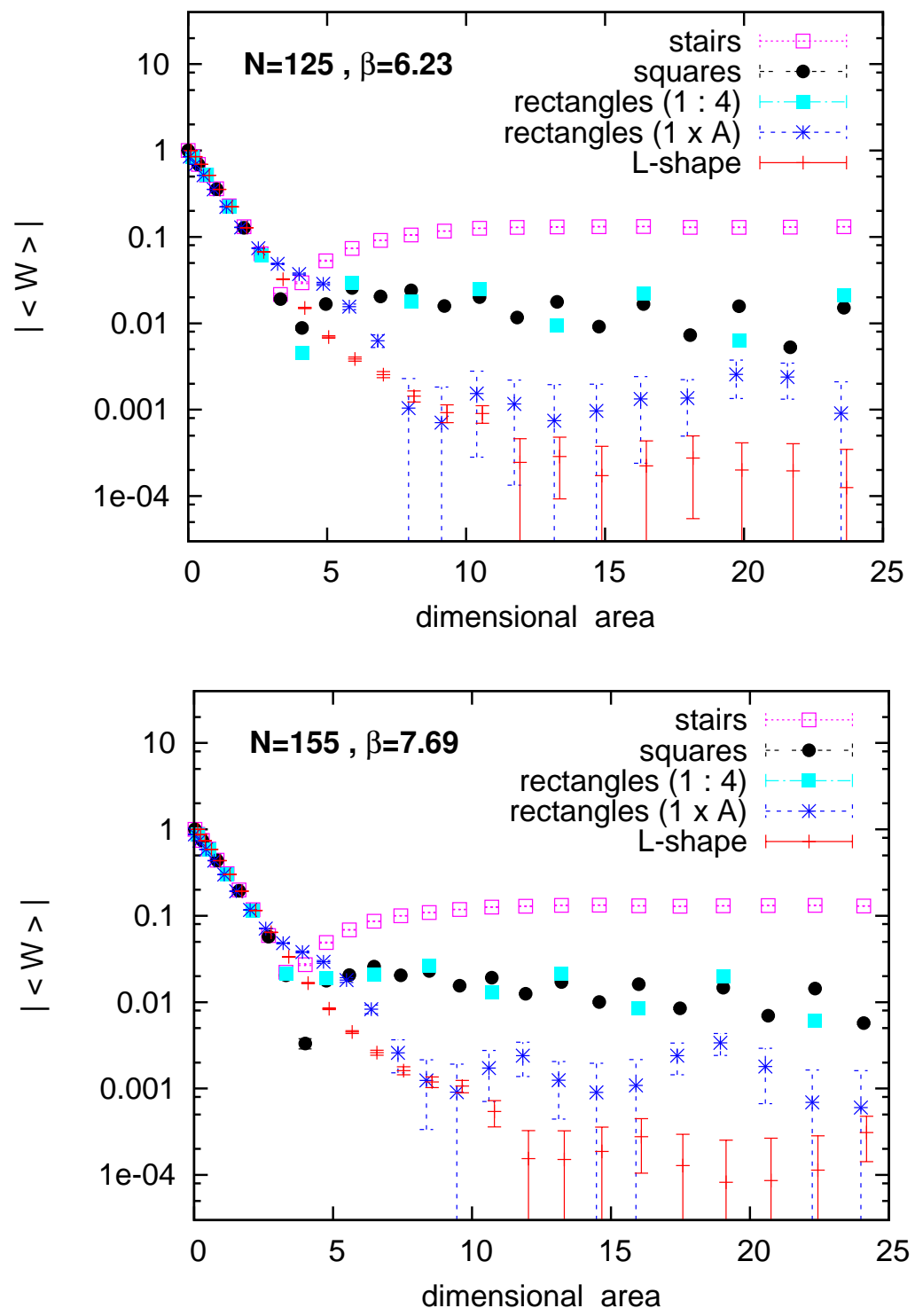

Figure 7: The absolute values of various Wilson loops at a fixed noncommutativity parameter $\theta=1.63$. On top we show results at $N=125$ and below at $N=155$. As we increase $N$ the dimensional volume grows and the lattice spacing shrinks, so that we approach simultaneously the limits to the continuum and to infinite volume (UV and IR limit). The striking similarity of these plots confirms that DSL convergence is reached. The results show a clear shape dependence beyond the area law regime, and therefore the breaking of APD symmetry. 

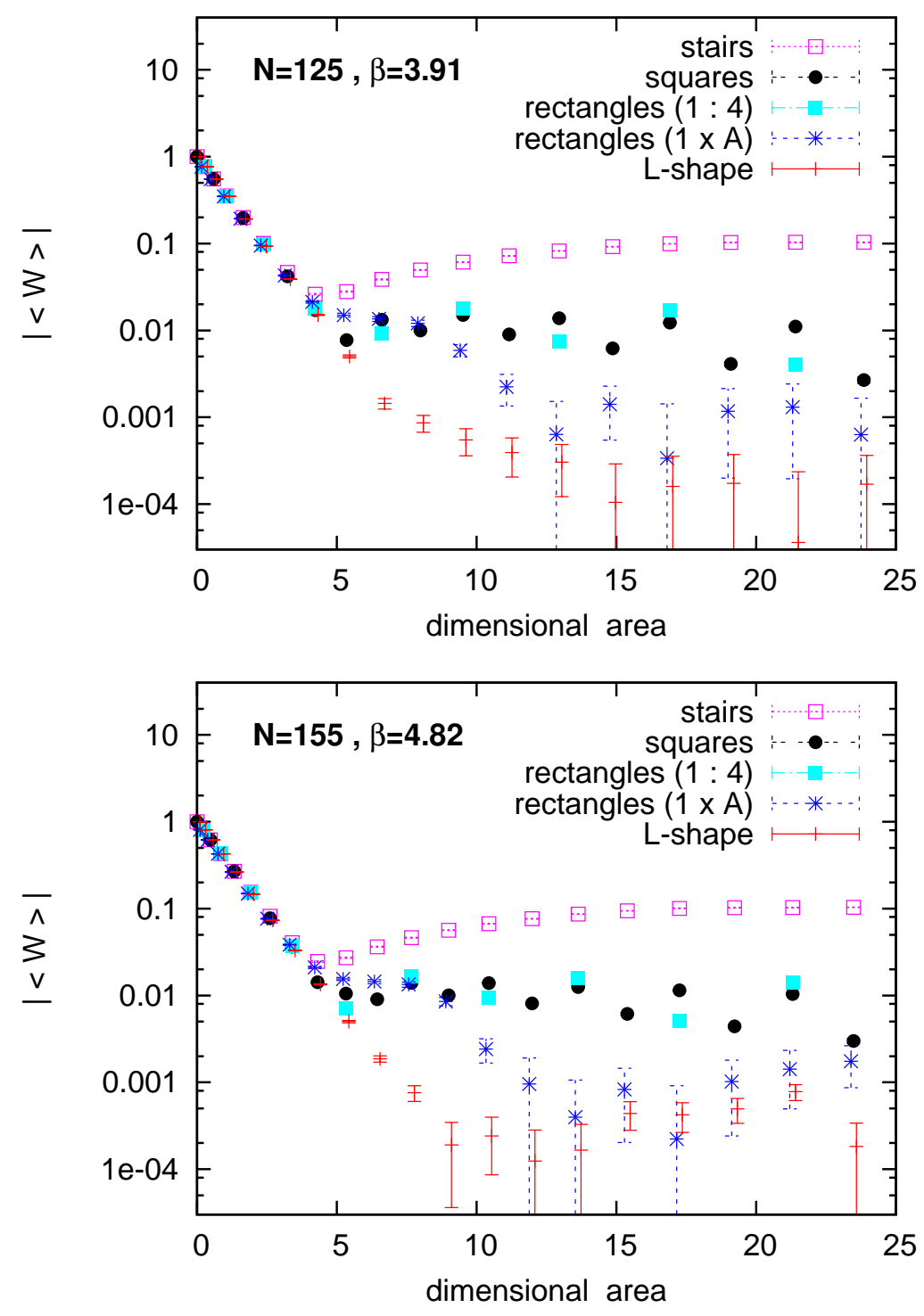

Figure 8: The analogous plots to Figure 7, but now at $\theta=2.63$. Again the results clearly confirm a shape dependence beyond the area law regime, which is stable as we increase $N$ towards the DSL.

$|\langle W\rangle|$ are sufficient to discard APD symmetry as a basic property of this theory. 

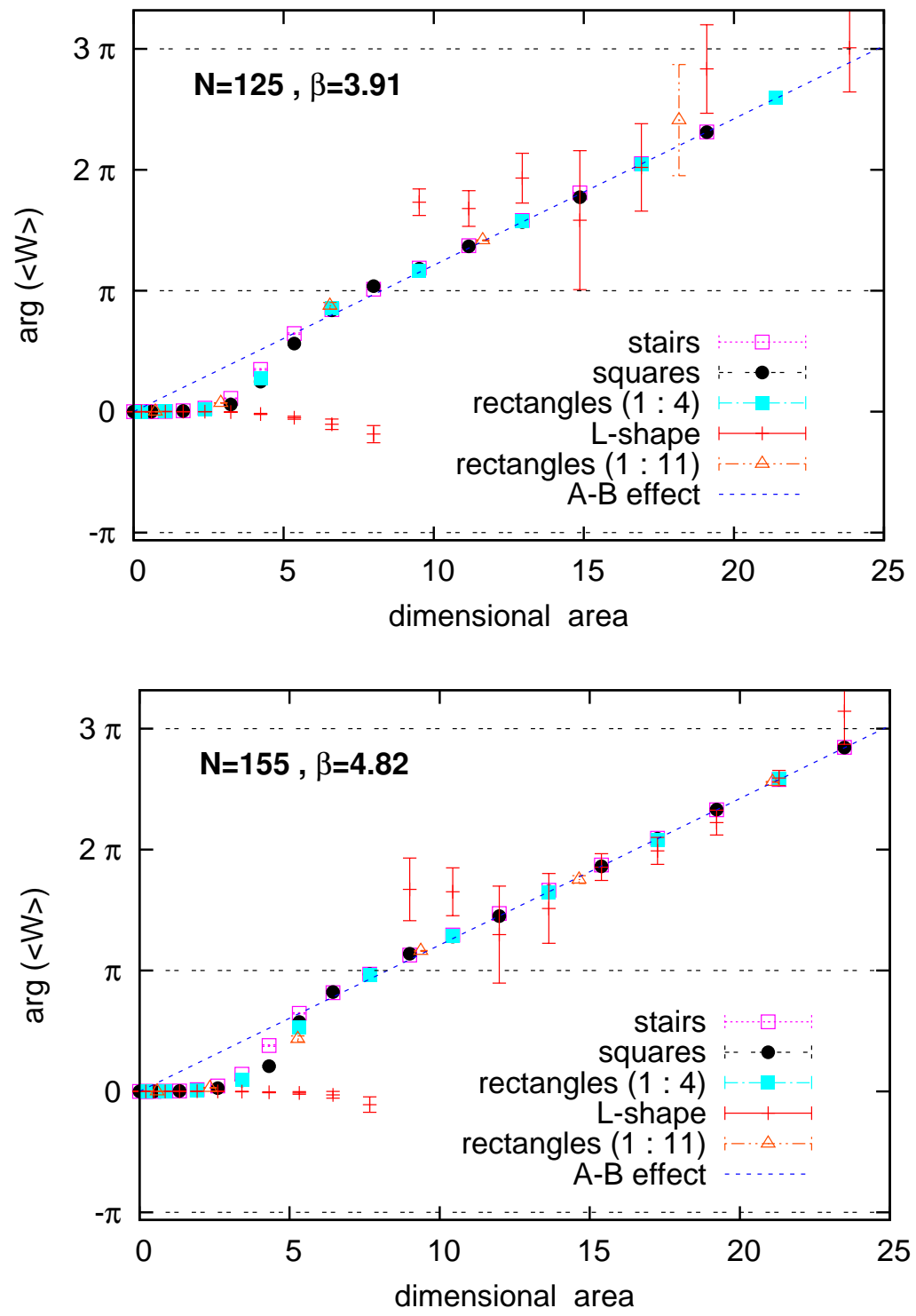

Figure 9: The phases of various Wilson loops at $\theta=2.63$ for $N=125$ (on top) and $N=155$ (below). The Aharonov-Bohm type behaviour in eq. (12) at large area is very well confirmed for the shapes shown here. They include results for rectangular loops with a fixed ratio of 4 and a ratio of 11 between the side lengths, so that its shape is invariant on the way to the DSL. The L-shape, on the other hand, becomes infinitesimally thin in this limit. Its phase follows eq. (12) with some fluctuations. 


\section{APD symmetry breaking for alternative ob- servables}

Our results for the standard observables $|\langle W\rangle|$ and $\arg (\langle W\rangle)$ were presented in Section 4 and illustrated the shape dependence of Wilson loops with the same area (in dimensional units). We gave evidence for this effect to persist in the DSL.

In this section we present the corresponding results for $\langle|W|\rangle$ and $\langle\arg (W)\rangle$, which are somewhat different observables. The quantities of Section 4 are tractable in perturbation theory, and these are therefore the observables that have been addressed in Refs. [8-14]. Moreover they were measured in the previous numerical study in Ref. [15]. On the other hand, the quantities of this section are generally not considered in analytic work. In the appendix we comment on the prospects of the perturbative treatment of $\langle|W|\rangle$. However, $\langle|W|\rangle$ and $\langle\arg (W)\rangle$ can be handled numerically without specific problems, and they also represent valid physical observables 9 For instance, in lattice gauge theory it is also usual to measure $\langle|P|\rangle$ ( $P$ being the Polyakov loop) its magnitude serves as a criterion to distinguish the phases of confinement and deconfinement.

We add these observables here in order to supply further strength to our observation of APD symmetry breaking. The new observables are suitable for this purpose, in particular because $\langle|W|\rangle$ does not become as tiny as $|\langle W\rangle|$ at moderate and large area, which leads to smaller relative errors.

We first present the results for $\langle|W|\rangle$ for two values of $N$ at $\theta=1.63$ (Figure 10) and at $\theta=2.63$ (Figure 11), in analogy to Figures 7 and 8 , The new observables are practically identical to those of Section 4 at small area, where the phase is tiny throughout the Monte Carlo history. They differ, however, as the area grows beyond this regime. There $\langle|W|\rangle$ is significantly larger, which enables a better distinction (without overlapping error bars). But the qualitative behaviour is very similar to Section 4.

Figure 12 shows the phases $\langle\arg (W)\rangle$ at the same parameters as in Figure 10. It can be compared to the phase $\arg (\langle W\rangle)$ presented before in Figure 9 (for $\theta=2.63$ ). Also this phase follows closely the Aharonov-Bohm type behaviour of eq. (12) for the squares, the stairs loops and for the rectangle with a fixed side ratios. (For the squares this behaviour was also observed in 4d NC U(1) gauge theory [19].) Again the phase of the L-shape loops deviates. It fluctuates strongly in the Monte Carlo history all over the interval $(-\pi, \pi]$, keeping its mean values close to zero.

\footnotetext{
${ }^{9}$ Actually we considered the observables of this section already in Section 3 when we discussed the validity of the scale identified from the planar limit (Figures 4 and 51).
} 

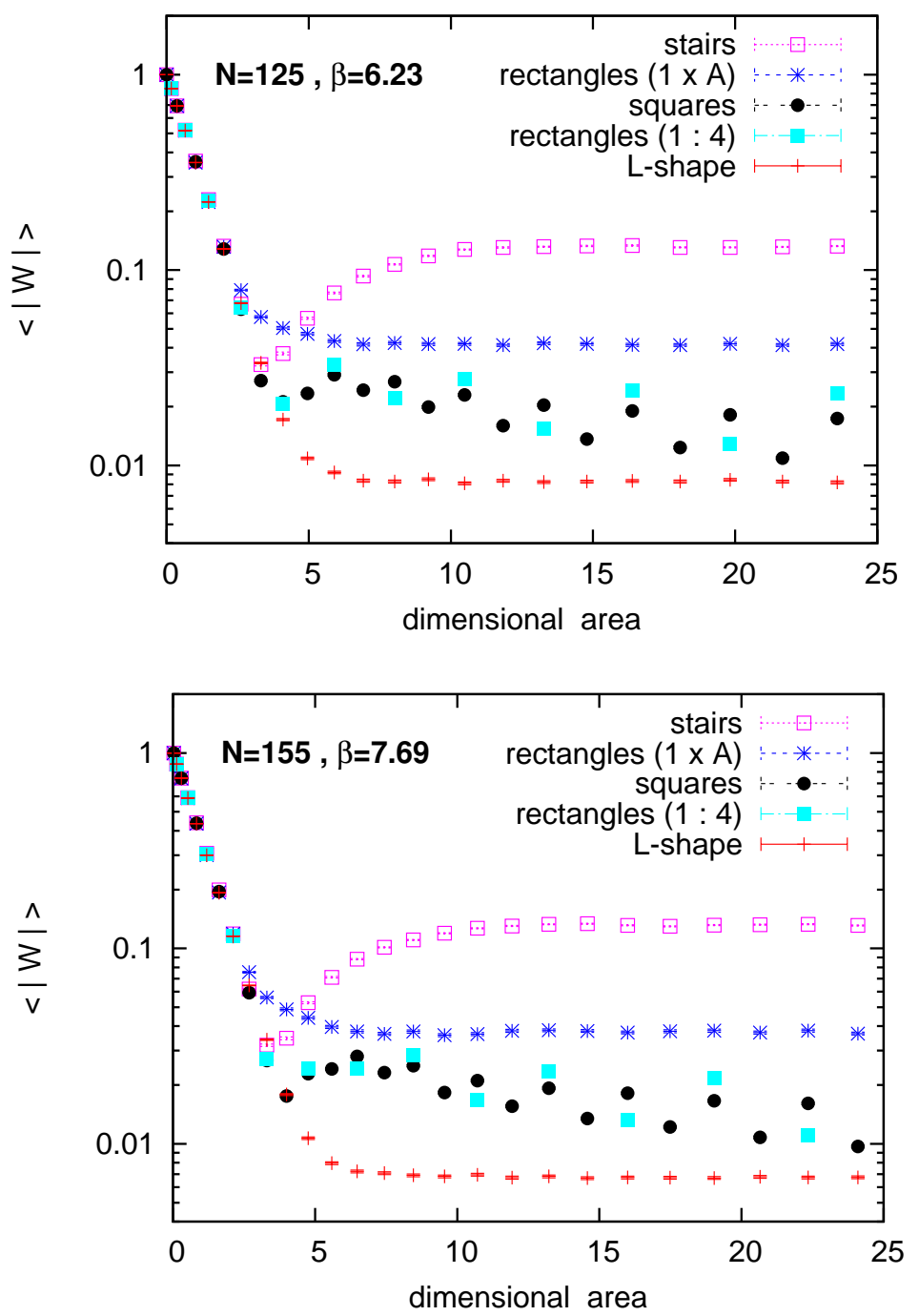

Figure 10: The expectation values $\langle|W|\rangle$ for various Wilson loops at a fixed non-commutativity parameter $\theta=1.63$. On top we show results at $N=125$ and below at $N=155$. The striking similarity of these plots confirms that $D S L$ convergence is reached. The results reveal a clear shape dependence beyond the area law regime also for this observable.

At last we consider the phases of the rectangles of the form $1 \times A$, i.e. of an infinitesimally narrow shape in the DSL. We mentioned in Section 4 that these phases do not follow eq. (12). In Figure 13 we add the phases for these rectangles at $\theta=2.62$. We show $\arg (\langle W\rangle)$ on the left, and $\langle\arg (W)\rangle$ 

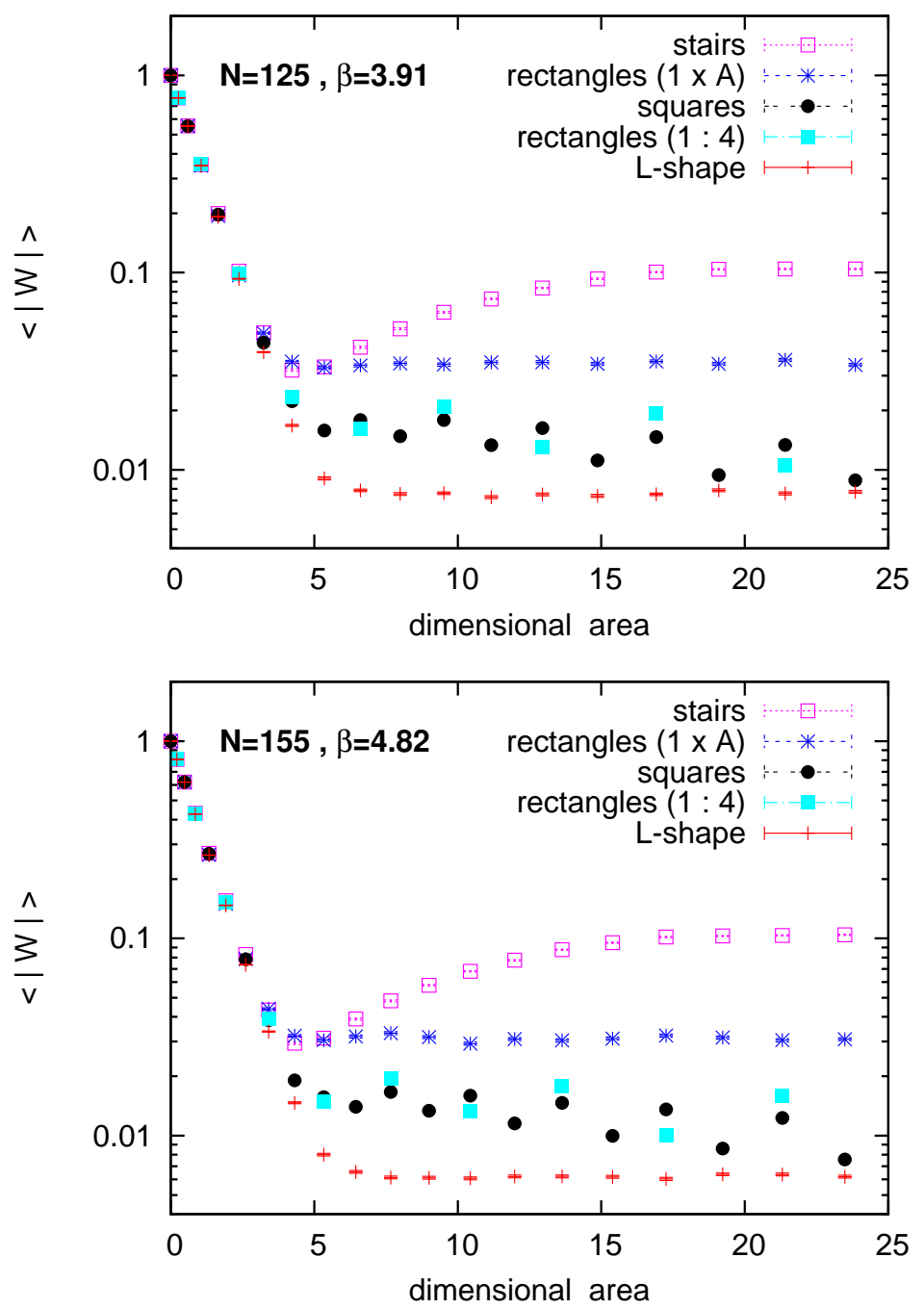

Figure 11: The analogous plots to Figure 10, but now at $\theta=2.63$. The results affirm once more a shape dependence beyond the area law regime, which is stable as we increase $N$. In particular the differences at fixed area do not shrink as we increase $N$ towards the DSL, in contrast to the planar limit behaviour in Figure 5 .

on the right. They are very similar, and - more importantly - they are in excellent agreement for the different $N$ values. This confirms once more that we access the double scaling window with the parameters used. 

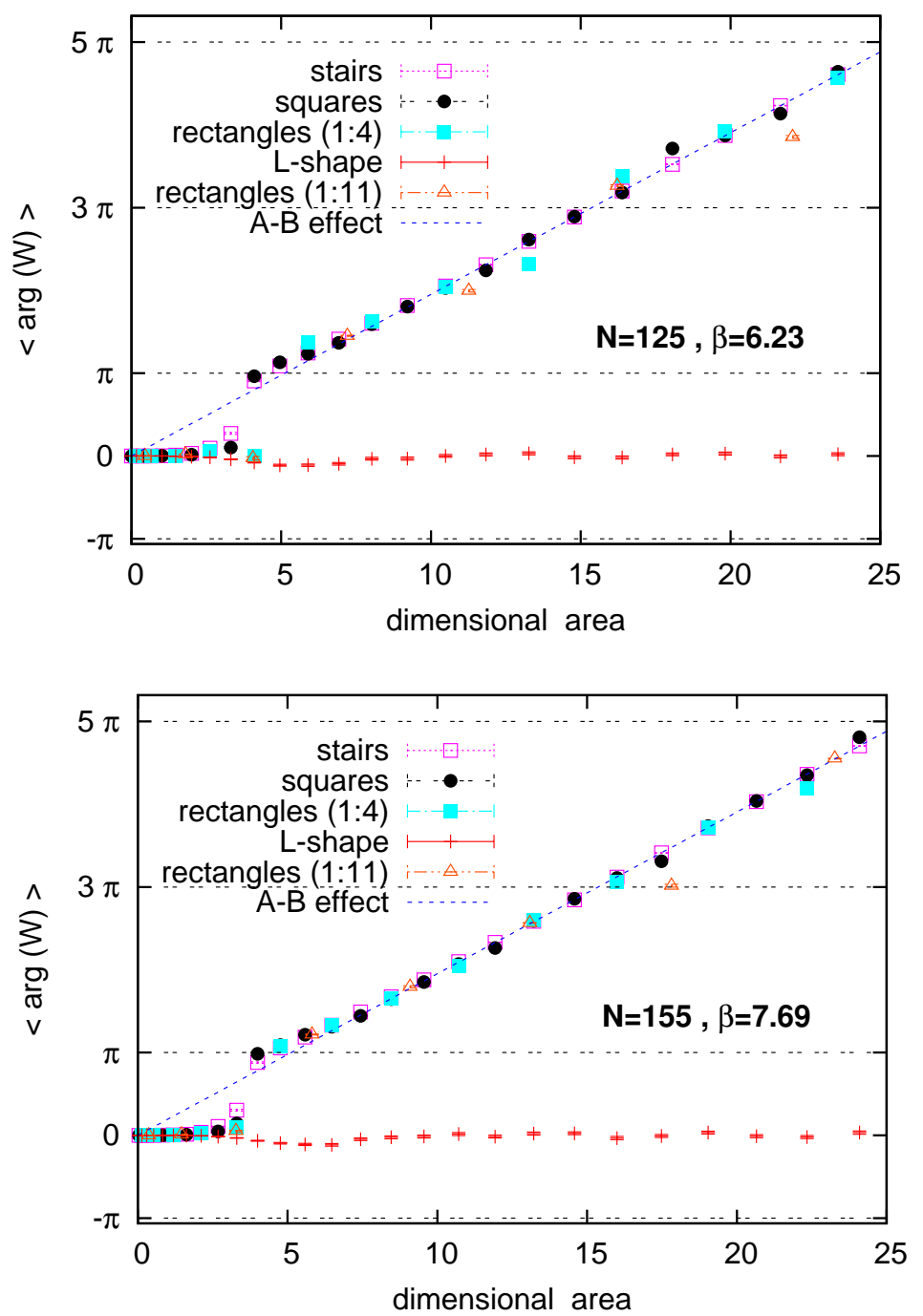

Figure 12: The phases $\langle\arg (W)\rangle$ of various Wilson loops at $\theta=1.63$ for $N=125$ (on top) and $N=155$ (below). The Aharonov-Bohm type behaviour in eq. (12) at large area holds well also for this phase for the stair loops, the squares and the rectangular loops with a fixed side ratios of 4 and of 11 . On the other hand, the average phase of the L-shape loops remains very small even at large areas.

This section presented additional APD symmetry breaking results, now for $\langle|W|\rangle$ and $\langle\arg (W)\rangle$. The values and physical meanings of these observables are different from those in Section 4, but qualitatively the results are in 

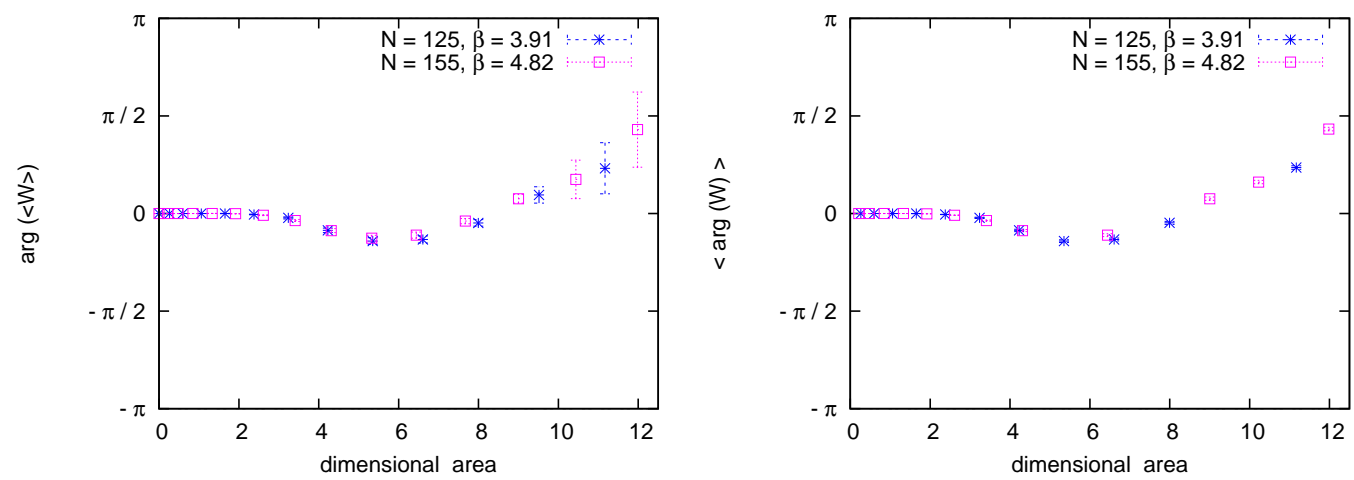

Figure 13: The phases of narrow rectangles with the shape $1 \times A$ at $\theta=2.62$. We show the phase of $\langle W\rangle$ (on the left) and the expectation value of $\arg (W)$ (on the right). Their values are very similar, but these phases are not related to eq. (12). The essential observation here is that the phases practically coincide for the two sets of parameters, which confirms again that we do see the window that extrapolates to the DSL.

full agreement. The observables added here provide further strength to our investigation of APD symmetry breaking — see also the theoretical reasoning in the Appendix.

\section{The $S L(2, R)$ symmetry breaking}

While the breaking of the full APD symmetry has already been demonstrated extensively, the specific case of the $S L(2, R)$ symmetry subgroup may seem less obvious from the Figures shown so far. Hence this section focuses on rectangles only to illustrate in particular the breaking of this subgroup.

Figures 14 and 15 are dedicated to the decay of the absolute values $|\langle W\rangle|$ and $\langle|W|\rangle$ for the rectangles. We show in both figures the behaviour for fixed $\theta$, where we include the data from $N=125$ and $N=155$ in the same plot. Here we also add results for the decay of rectangles with a fixed side ratio of 11 , which are helpful to demonstrate the $S L(2, R)$ breaking more clearly.

For the rectangles with fixed shapes these observables tend to oscillate as we vary the dimensional area beyond the area law regime. This observation is well compatible with the data from both $N$ values, hence it seems to characterise the DSL. These oscillations have similar mean values, but the amplitude is significantly larger for the ratio 11 between the side length when compared to ratio 4 or 1 (squares).

We conclude that the $S L(2, R)$ symmetry is indeed more viable to some approximation than the rest of the APD symmetry group, but its breaking 
is nevertheless manifest on the non-perturbative level.
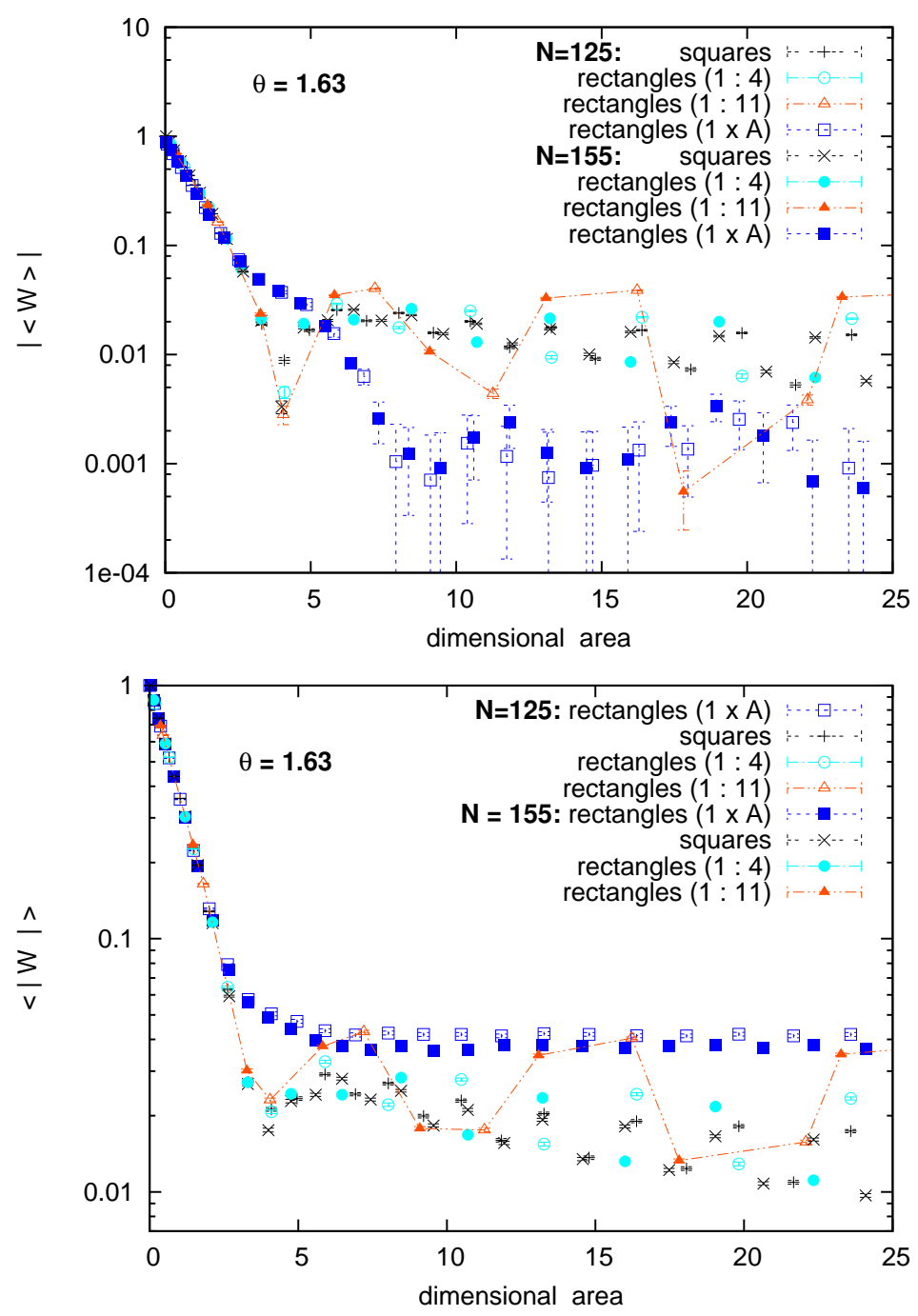

Figure 14: The decay of the absolute values $|\langle W\rangle|$ (on top) and of $\langle|W|\rangle$ (below) for rectangular loops at $\theta=1.63$. Beyond the area law regime, the rectangles with finite sides in the DSL (i.e. with a fixed side ratio) tend to oscillate around $\approx 0.01$ for $|\langle W\rangle|$ and a somewhat larger value for $\langle|W|\rangle$. The amplitudes, however, depend clearly on the side ratio, which shows the $S L(2, R)$ symmetry breaking. This is most evident for the rectangles with side ratio 11 (their data are connected by a line to guide the eye). 

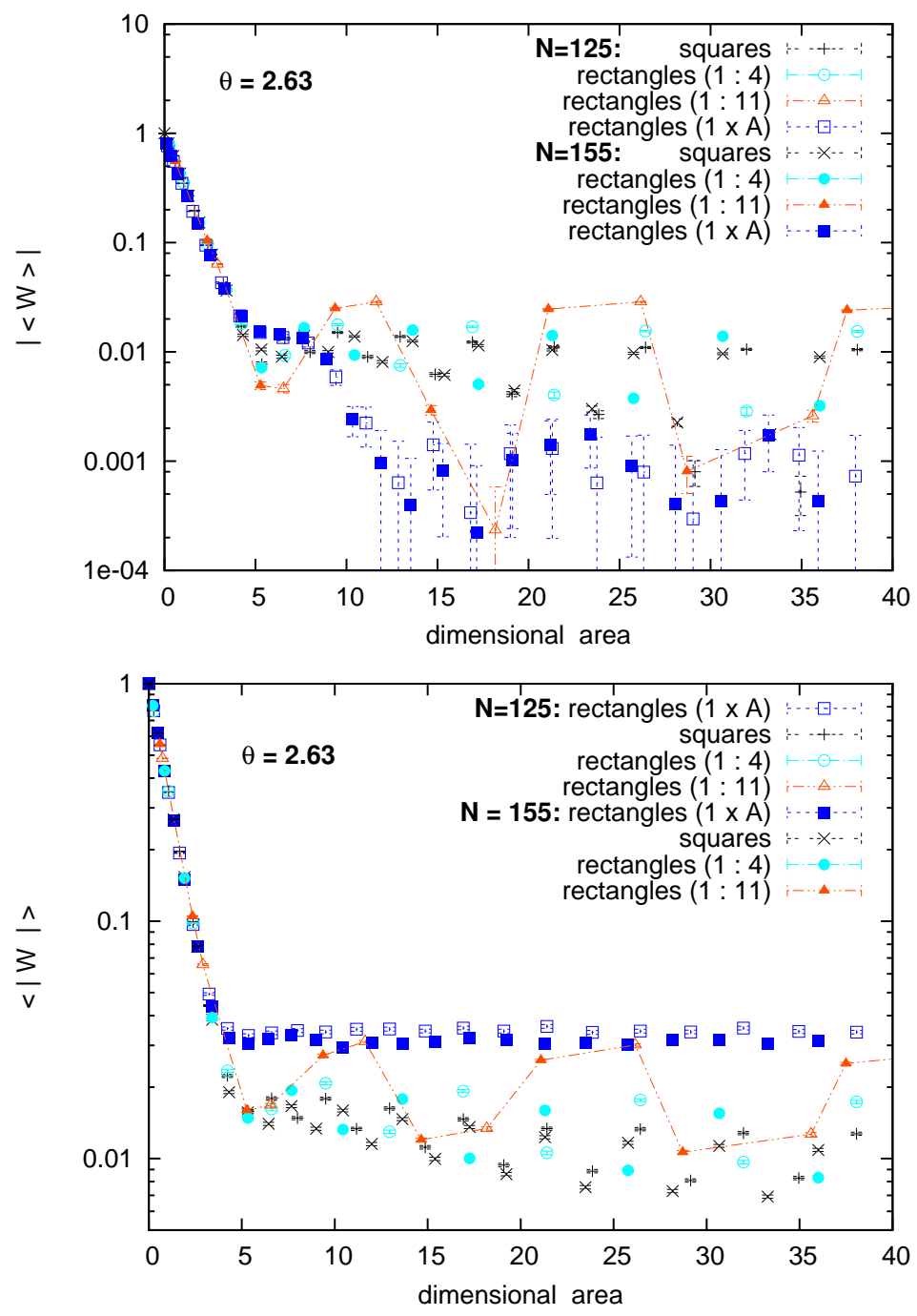

Figure 15: The analogous plots to Figure 14, but now at $\theta=2.63$. The observed decays of the rectangular Wilson loops is very similar for both noncommutativity parameters $\theta$ that we consider. Again the $S L(2, R)$ symmetry breaking can been seen best from the data for rectangles with side ratio 11 (which are connected to guide the eye).

\section{Conclusions}

While APD symmetry holds in ordinary two-dimensional Yang-Mills theory, it turns out to be broken by non-commutativity. Perturbation theory can reveal this effect, depending on the order considered. Since this is still re- 
strictive, to a finite order the symmetry may be protected in part [11], with a residual subgroup showing up in other approaches as well [13].

However, on the non-perturbative level and by considering finite $\theta$, the quantum effects unfold their full power and destroy the APD symmetry, including the subgroup $S L(2, R)$. Here we presented explicit results for the APD symmetry breaking. We considered four types of Wilson loops with polygonal contours, without multiple-intersections. At the same area, numerical simulations revealed the shape dependence of the Wilson loop expectation values on the lattice. Our results were obtained for constant non-commutativity parameters $\theta$ at different values of $N$ - for increasing $N$ the volume becomes larger (in dimensional units) and the lattice finer. The results for the Wilson loops remain very stable, hence they allow for a reliable extrapolation to a continuous plane of infinite extent at fixed non-commutativity (Double Scaling Limit). This limit reveals the breaking of APD symmetry - including the $S L(2, R)$ subgroup - on the nonperturbative level. Furthermore our results extend the loss of APD symmetry to the discretised model in a finite volume.

To be more precise, the APD symmetry holds very well for relatively small loop areas (in dimensional units). For a fixed lattice area this corresponds to the weak coupling regime, and hence the agreement with the leading order perturbative results appears consistent. However, as the dimensional area increases, this agreement - and therefore the APD symmetry - collapses: it partially persists in the phase of the Wilson loops, but not in their absolute values. We also observed that the $S L(2, R)$ subgroup has is a relatively high viability as an approximate symmetry (which is consistent with perturbative results), but its breaking becomes manifest for strongly anisotropic transformations in this subgroup.

We conclude that NC gauge theory has a rich structure, even in $d=2$, far beyond its commutative counterpart. This conclusion rises hope for exciting effects. On the other hand, it also means that an analytical solution is unlikely. Therefore numerical results are of great importance, as it is the case in commutative Yang-Mills theories in four dimensions.

Acknowledgements: We are indebted to F. Hofheinz and J. Volkholz for valuable help with the numerical work, and to A. Bassetto for reading the manuscript. We also thank them, as well as G. De Pol, H. Dorn, L. Griguolo, J. Nishimura, P. Sodano, Y. Susaki and F. Vian for interesting discussions. The work of A.B. was supported by Istituto Nazionale di Fisica Nucleare (INFN), by the Deutsche Forschungsgemeinschaft (DFG), and by the Pan-European Research Infrastructure on High Performance Computing (HPC-Europa). He thanks M. Müller-Preußker for kindly supporting his HPC-Europa application. The work of A.T. was sup- 
ported by the DFG within the Schwerpunktprogramm Stringtheorie 1096, by INFN with a Bruno Rossi postdoctoral fellowship, and by the U.S. Department of Energy (D.O.E.) under cooperative research agreement DE-FG02-05ER41360. The computations were performed on the Hochleistungs-Rechenzentrum in Stuttgart (HLRS) and on a PC cluster at Humboldt-Universität.

\section{A The perturbative treatment of $\langle|W|\rangle$}

In this appendix we comment on the perturbative treatment of the observable $\langle|W|\rangle$, along the lines of the formalism constructed in Refs. [8,9]. We refer the reader to those works for the details and the relevant literature, while here we just recall the basic ingredients needed for our argument.

A convenient gauge choice for perturbative Wilson loop calculations is the light-cone gauge $A_{-}=0$. Faddeev-Popov ghosts are known to decouple also in the $\mathrm{NC}$ case, and the two-dimensional Lagrangian in this gauge looks indeed free. The non-trivial information on the dynamics is encoded in the singular behaviour of the propagator

$$
D_{++}=\mathrm{i}\left[k_{-}^{-2}\right] .
$$

Two prescriptions for the pole are important, the Cauchy principal value method by 't Hooft, and the prescription due to $\mathrm{Wu}$, Mandelstam and Leibbrandt [35], which employs the propagator

$$
D_{++}=\mathrm{i}\left[k_{-}+\mathrm{i} \epsilon k_{+}\right]^{-2} .
$$

In the commutative case, the latter prescription is genuinely perturbative, while the former is able to produce results for the Wilson loops, which take into account non-perturbative contributions, and it yields exponentiation (area law). But in the NC case, the former prescription behaves very wildly and has been soon abandoned, while the latter gives sensible results at all the orders considered. Also, the rotation to the Euclidean version of the theory turned out to be useful.

When examining the perturbative series of the Wilson loop $\langle W\rangle$, one notices that the NC phase factor intermingles non-trivially, in the non-planar diagrams, with the propagators in the momentum and contour integrations. The results are themselves expansions, typically in $1 / \theta$; the Wilson loop is analytic around $\theta=\infty$.

The perturbative series for the observable $\langle|W|\rangle$ looks a priori quite dif- 
ferent from the one of $|\langle W\rangle|$. The relevant expansion in the $U(1)$ case reads

$$
\begin{aligned}
\langle|W|\rangle= & \langle| \sum_{n=0}^{\infty}(\mathrm{i} g)^{n} \int_{0}^{1} d s_{1} \ldots \int_{s_{n-1}}^{1} d s_{n} \dot{\xi}_{-}\left(s_{1}\right) \ldots \dot{\xi}_{-}\left(s_{n}\right) \\
& \int d^{2} x A_{+}\left(x+\xi\left(s_{1}\right)\right) \star \ldots \star A_{+}\left(x+\xi\left(s_{n}\right)\right)|\rangle .
\end{aligned}
$$

Clearly the presence of the modulus inside the quantum average complicates the task of rearranging the expansion in terms of the Green functions of the theory, for one has first to write it schematically as $\sqrt{\left(\sum \ldots\right)\left(\sum \ldots\right)^{*}}$ and then perform a Taylor series in $g$. The fact that the sums begin with a constant, followed by terms with an increasing number of fields, allows to formally treat the series as analytic around $g=0$. At the end, one computes the vacuum expectation value, based on the Green functions

$$
\left\langle 0\left|\mathcal{T} A_{+}\left(x+\xi\left(s_{1}\right)\right) \star \ldots \star A_{+}\left(x+\xi\left(s_{2 n}\right)\right)\right| 0\right\rangle .
$$

Each term can in principle be treated similarly to the standard case: one is left with multiple integrals of propagators and Moyal phases, in momentum space and on domains which are simplexes in the contour parameters. These contributions can be visualised by attaching the propagator lines along the contour itself: different simplexes correspond then to different crossing patterns of the propagators.

In the simplest case of the $\mathcal{O}\left(g^{4}\right)$ computation, the momentum integrals can be carried out exactly using complex plane techniques and identities of Bessel functions, and the remaining contour integration is then performed numerically. For the new observable, care must be taken in computing some of the integrals, because one encounters terms like

$$
\left\langle\left(\int_{0}^{1} d s_{i} \dot{\xi}_{-}\left(s_{i}\right) \int d^{2} x \exp \left[\mathrm{i} p\left(x+\xi\left(s_{i}\right)\right)\right] A_{+}(p)\right)^{2}\right\rangle,
$$

which appear already at the order $\mathcal{O}\left(g^{2}\right)$. This order is unaffected by the Moyal phase, but still the overall integration over the space-time base-point $x$ forces the appearance of a correlator $\left\langle A_{+}(0) A_{+}(0)\right\rangle$ at zero momentum which is infrared divergent. On the other hand, the integration over the closed contour vanishes because it reduces to $\int_{0}^{1} d s_{i} \dot{\xi}_{-}\left(s_{i}\right)=0$. Therefore, one has to fix the order of integrations, such that the contour integration is performed first. Still, it is not clear if this can be made consistent at any order, or if other potentially divergent terms will appear due to the nesting of contour integrals, or, most important, due to interference with the NC phase factor. We leave this interesting analysis for future developments. 
Here we would like to remark that, provided one can consistently treat these ambiguities, then the arguments about order-by-order APD invariance at $\theta=0$ seem to apply. This implies that the observed shape-dependence of $\langle|W|\rangle$ in the NC plane appears as a valid argument for concluding that APD symmetry breaks down when going from $\theta=0$ to a finite $\theta$ value.

\section{References}

[1] E. Witten, Commun. Math. Phys. 141 (1991) 153; J. Geom. Phys. 9 (1992) 303.

[2] A.A. Migdal, Sov. Phys. JETP 42 (1975) 413 [Zh. Eksp. Teor. Fiz. 69 (1975) 810].

[3] B.E. Rusakov, Mod. Phys. Lett. A5 (1990) 693; Phys. Lett. B303 (1993) 95. M.R. Douglas and V.A. Kazakov, Phys. Lett. B319 (1993) 219. D.V. Boulatov, Mod. Phys. Lett. A9 (1994) 365. J.M. Daul and V.A. Kazakov, Phys. Lett. B335 (1994) 371.

[4] Y. Makeenko and A.A. Migdal, Phys. Lett. B88 (1979) 135 (Erratumibid. B89 (1980) 437). V.A. Kazakov and I.K. Kostov, Nucl. Phys. B176 (1980) 199; Phys. Lett. B105 (1981) 453. V.A. Kazakov, Nucl. Phys. B179 (1981) 283.

[5] F. Lizzi, R.J. Szabo and A. Zampini, JHEP 0108 (2001) 032

[6] M.M. Sheikh-Jabbari, JHEP 9906 (1999) 015.

[7] L.D. Paniak and R.J. Szabo, JHEP 0305 (2003) 029.

[8] A. Bassetto, G. Nardelli and A. Torrielli, Nucl. Phys. B617 (2001) 308.

[9] A. Bassetto, G. Nardelli and A. Torrielli, Phys. Rev. D66 (2002) 085012. A. Torrielli, "Noncommutative perturbative quantum field theory: Wilson loop in two-dimensional Yang-Mills, and unitarity from string theory", Ph.D. Thesis, Padua (2002) [hep-th/0301091].

[10] J. Ambjørn, A. Dubin and Y. Makeenko, JHEP 0407 (2004) 044.

[11] A. Bassetto, G. De Pol, A. Torrielli and F. Vian, JHEP 05 (2005) 061.

[12] M. Cirafici, L. Griguolo, D. Seminara and R.J. Szabo, JHEP 0510 (2005) 030. 
[13] M. Riccardi and R.J. Szabo, hep-th/0701273.

[14] J. Ambjørn, A. Dubin and Y. Makeenko, hep-th/0703123.

[15] W. Bietenholz, F. Hofheinz and J. Nishimura, JHEP 09 (2002) 9. F. Hofheinz, "Field theory on a noncommutative plane: A Nonperturbative study", Ph.D. Thesis, Berlin (2003) [hep-th/0403117].

[16] R.J. Szabo, Int. J. Mod. Phys. A19 (2004) 1837.

[17] N. Seiberg and E. Witten, JHEP 09 (1999) 032.

[18] R.J. Szabo, Phys. Rep. 378 (2003) 207.

[19] W. Bietenholz, J. Nishimura, Y. Susaki and J. Volkholz, JHEP 10 (2006) 042. J. Volkholz, "Nonperturbative Studies of Quantum Field Theories on Noncommutative Spaces", Ph.D. Thesis, Berlin (2007).

[20] S. Minwalla, M. van Raamsdonk and N. Seiberg, JHEP 02 (2000) 020.

[21] W. Bietenholz, F. Hofheinz and J. Nishimura, JHEP 0405 (2004) 047.

[22] A. González-Arroyo and M. Okawa, Phys. Rev. 27D (1983) 2397. A. González-Arroyo and C.P. Korthals Altes, Phys. Lett. B131 (1983) 396.

[23] H. Aoki, N. Ishibashi, S. Iso, H. Kawai, Y. Kitazawa and T. Tada, Nucl. Phys. B565 (2000) 176.

[24] J. Ambjørn, Y. Makeenko, J. Nishimura and R.J. Szabo, JHEP 11 (1999) 29; Phys. Lett. B480 (2000) 399; JHEP 05 (2000) 023.

[25] N. Ishibashi, S. Iso, H. Kawai and Y. Kitazawa, Nucl. Phys. B573 (2000) 573.

[26] D.J. Gross, A. Hashimoto and N. Itzhaki, Adv. Theor. Math. Phys. 4 (2000) 893. L. Alvarez-Gaumé and S.R. Wadia, Phys. Lett. B501 (2001) 319.

[27] K. Fabricius and O. Haan, Phys. Lett. 143B (1984) 459.

[28] D.J. Gross and E. Witten, Phys. Rev. D21 (1980) 446.

[29] T. Eguchi and H. Kawai, Phys. Rev. Lett. 48 (1982) 1063.

[30] T. Nakajima and J. Nishimura, Nucl. Phys. B528 (1998) 355. 
[31] W. Bietenholz, A. Bigarini, F. Hofheinz, J. Nishimura, Y. Susaki and J. Volkholz, Fortsch. Phys. 53 (2005) 418.

[32] A. Bigarini, "Numerical Simulations of 2d non-commutative field theories", Ph.D. Thesis, Perugia (2005).

[33] S.S. Gubser and S.L. Sondhi, Nucl. Phys. B605 (2001) 395. G.-H. Chen and Y.-S. Wu, Nucl. Phys. B622 (2002) 189. P. Castorina and D. Zappalà, Phys. Rev. D68 (2003) 065008.

[34] J. Ambjørn and S. Catterall, Phys. Lett. B549 (2002) 253. W. Bietenholz, F. Hofheinz and J. Nishimura, JHEP 06 (2004) 42.

[35] T.T. Wu, Phys. Lett. B71 (1977) 142. S. Mandelstam, Nucl. Phys. B213 (1983) 149. G. Leibbrandt, Phys. Rev. D29 (1984) 1699. 